\title{
Prognostic Value of Neoadjuvant Chemotherapy in Locoregionally Advanced Nasopharyngeal Carcinoma with Low Pre-treatment Epstein-Barr Virus DNA: a Propensity-matched Analysis
}

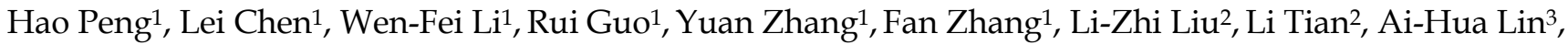 \\ Ying Sun ${ }^{1}$, Jun $\mathrm{Ma}^{1}$ \\ 1. Department of Radiation Oncology, Sun Yat-sen University Cancer Center, State Key Laboratory of Oncology in Southern China, Collaborative Innovation \\ Center for Cancer Medicine, People's Republic of China. \\ 2. Imaging Diagnosis and Interventional Center, Sun Yat-sen University Cancer Center, State Key Laboratory of Oncology in Southern China, Collaborative \\ Innovation Center for Cancer Medicine, People's Republic of China \\ 3. Department of Medical Statistics and Epidemiology, School of Public Health, Sun Yat-sen University, People's Republic of China. \\ $\triangle$ Corresponding author: Jun Ma, MD, State Key Laboratory of Oncology in South China, Department of Radiation Oncology, Sun Yat-sen University Cancer \\ Center, 651 Dongfeng Road East, Guangzhou 510060, People's Republic of China. Telephone: +86-20-87343469 Fax: +86-20-87343295 E-mail: \\ majun2@mail.sysu.edu.cn.
}

(1) Ivyspring International Publisher. Reproduction is permitted for personal, noncommercial use, provided that the article is in whole, unmodified, and properly cited. See http://ivyspring.com/terms for terms and conditions.

Received: 2016.04.04; Accepted: 2016.05.24; Published: 2016.07.05

\begin{abstract}
Background: The aim of this study is to investigate the prognostic value of neoadjuvant chemotherapy (NCT) in locoregionally advanced nasopharyngeal carcinoma (NPC) with low pre-treatment Epstein-Barr virus (EBV) DNA in the era of intensity-modulated radiotherapy (IMRT).

Methods: Data on 1099 locoregionally advanced NPC patients treated with IMRT were retrospectively reviewed. Propensity score matching (PSM) method was adopted to balance influence of covariates. Patient survival between NCT and non-NCT groups were compared.

Results: The cut-off value of pre-treatment Epstein-Barr virus DNA (pre-DNA) was 1550 copies $/ \mathrm{ml}$ for DMFS (area under curve [AUC], 0.655; sensitivity, 0.819; specificity, 0.445). For the 145 pairs selected by PSM, the 3-year distant metastasis-free survival (DMFS), overall survival (OS), disease-free survival (DFS) and locoregional relapse-free survival (LRRFS) rates were $98.6 \%$ vs. $93.7 \%(P=0.101)$, $95.8 \%$ vs. $94.4 \%(P=0.881), 91.7 \%$ vs. $87.5 \%(P=0.309)$ and $94.4 \%$ vs. $95.0 \%(P=0.667)$, respectively. Multivariate analysis did not identify NCT as an independent prognostic factor $(P>0.05$ for all rates), and stratified analysis based on overall stage (III and IV) and N category (N0-1 and N2-3) also got the same results.

Conclusion: NCT was not established as an independent prognostic factor, and it should not be used in locoregionally advanced NPC with low pre-DNA.
\end{abstract}

Key words: nasopharyngeal carcinoma; neoadjuvant chemotherapy; Epstein-Barr virus DNA; prognosis.

\section{Introduction}

Nasopharyngeal carcinoma (NPC) is different from other head and neck cancers by its epidemiology, clinical characteristics and methods of treatment. The worldwide distribution of NPC is extremely unbalanced, with an age-standard incidence rate of $20-50$ per 100,000 males in south China to 0.5 per 100,000 in mainly white populations
[1]. Radiotherapy (RT) has been the primary treatment for NPC because of anatomic constraints and its high degree of radiosensitivity. NPC is also a chemosensitive tumor, and many trials have proven that a combination of radiotherapy and chemotherapy is better than radiotherapy alone [2-5]. Therefore, concurrent chemoradiotherapy (CCRT) has been 
established as the main treatment for locoregionally advanced NPC by National Comprehensive Cancer Network (NCCN) guidelines.

In the era of intensity-modulated radiotherapy (IMRT), distant metastasis has been the main failure pattern [6]. Therefore, for the last decade, most attention [7-12] has been focused on neoadjuvant chemotherapy (NCT) as it may reduce distant metastasis and improve overall survival. However, the results from these trials were controversial. Recently, a meta-analysis by Ouyang et al.[13] revealed NCT could effectively enhance overall survival and reduce distant metastasis rate.

Epstein-Barr virus (EBV) DNA has been proven to be a reliable biomarker in predicting prognosis [14-18]. Our previous study revealed patients with undetectable pre-treatment EBV DNA (pre-DNA) had obviously better distant metastasis-free survival compared with patients with positive pre-DNA [19], which indicated that distant metastasis may not be the main failure pattern in advanced NPC with undetectable or low pre-DNA load. Moreover, Du et al. [20] also defined high-risk patients with locally advanced NPC who may benefit from NCT by using pre-DNA. Therefore, it is reasonable to speculate that NCT may be useless in advanced NPC with low pre-DNA. However, no relative study has investigated this relationship. Hence, we conducted this retrospective study to evaluate the value of NCT in locoregionally advanced NPC with low pre-DNA. To balance the influence of covariates, propensity score matching (PSM) method was adopted to compare survival outcomes and decrease potential bias [21].

\section{Materials and Methods}

\section{Patients Selection}

We retrospectively analysed the data on 1811 patients with newly diagnosed stage I-IVB NPC, who were treated between November 2009 and February 2012 at Sun Yat-sen university cancer center. The eligibility criteria of this study were as follows: (1) stage III-IVB NPC; (2) World Health Organization (WHO) pathology type II/III; (3) with the data of pre-DNA; (4) age 18 years or older. In total, 1099 $(60.7 \%)$ patients were recruited for the current study. This study was approved by the Research Ethics Committee of Sun Yat-sen university cancer center. Informed consent was obtained from all the patients.

\section{Clinical Staging}

The routine staging workup included a complete history and clinical examinations of the head and neck region, direct fibre-optic nasopharyngoscopy, magnetic resonance imaging (MRI) scans of the skull base and whole neck, chest radiography, whole-body bone scan and abdominal sonography, as well as positron emission tomography (PET)-CT. Tumour-related marker such as plasma EBV DNA load was quantified. All patients received a dental evaluation before radiotherapy.

All patients were restaged according to the $7^{\text {th }}$ edition of the International Union against Cancer/American Joint Committee on Cancer (UICC/AJCC) system [22]. All MRI materials and clinical records were reviewed separately by two radiologists (L.Z.L. and L.T.) employed at our hospital to minimize heterogeneity in restaging, and disagreements were resolved by consensus.

\section{Real-time quantitative EBV DNA PCR}

Measurement of the plasma EBV DNA load was performed before treatment, and plasma DNA was extracted and assayed using real-time quantitative PCR which was described previously [23]. The real-time quantitative PCR system was developed for plasma EBV DNA detection, and targeted the BamHI-W region of the EBV genome using primers 5'-GCCAGAGGTAAGTGGACTTT-3' and 5'-TACCA CCTCCTCTTCTTGCT-3'. The dual fluorescencelabelled oligomer 5'-(FAM)CACACCCAGGC ACACACTACACAT(TAMRA)-3' served as a probe. Sequence data for the EBV genome were obtained from the GeneBank sequence database. The plasma EBV DNA concentration was calculated using the following equation: $C=Q \times\left(V_{D N A} / V_{P C R}\right) \times\left(1 / V_{E X T}\right)$, in which $C$ represents the target concentration in plasma (copies/ml), $Q$ represents the target quantity (copy number) determined by PCR, $V_{D N A}$ represents the total volume of DNA obtained after extraction (typically $50 \mu \mathrm{l} /$ Qiagen extraction), $V_{P C R}$ represents the volume of DNA solution used for PCR (typically 2 $\mu \mathrm{l})$ and VEXT represents the volume of plasma extracted (typically $0.5 \mathrm{ml}$ ) [23].

\section{Clinical treatment}

\section{Radiotherapy}

All patients received IMRT at Sun Yat-sen university cancer center. A high-resolution planning computed tomography scan with contrast was taken from the vertex to $2 \mathrm{~cm}$ below the sternoclavicular joint at a slice thickness of $3 \mathrm{~mm}$. Target volumes were delineated slice-by-slice on treatment planning CT scans using an individualized delineation protocol that complies with International Commission on Radiation Units and Measurements reports 50 and 62. The prescribed doses were 66-72 Gy at 2.12-2.43 $\mathrm{Gy} /$ fraction to the planning target volume (PTV) of the primary gross tumour volume (GTVnx), 64-70 Gy to the PTV of the GTV of the involved lymph nodes 
(GTVnd), 60-63 Gy to the PTV of the high-risk clinical target volume (CTV1), and 54-56 Gy to the PTV of the low-risk clinical target volume (CTV2). All targets were treated simultaneously using the simultaneous integrated boost technique.

\section{Chemotherapy}

According to institutional guidelines, we recommended RT alone for stage I, CCRT for stage II, and CCRT +/- NCT/adjuvant chemotherapy (ACT) for stage III to IVA-B. NCT consisted of cisplatin (80 $\left.\mathrm{mg} / \mathrm{m}^{2}\right)$ with 5-fluorouracil $\left(1000 \mathrm{mg} / \mathrm{m}^{2}\right) \quad(\mathrm{PF})$, cisplatin $\left(75 \mathrm{mg} / \mathrm{m}^{2}\right)$ with docetaxel $\left(75 \mathrm{mg} / \mathrm{m}^{2}\right)(\mathrm{TP})$, or cisplatin $\left(60 \mathrm{mg} / \mathrm{m}^{2}\right)$ with 5-fluorouracil $(600$ $\left.\mathrm{mg} / \mathrm{m}^{2}\right)$ and docetaxel $\left(60 \mathrm{mg} / \mathrm{m}^{2}\right)(\mathrm{TPF})$ every three weeks for two or more cycles. Concurrent chemotherapy was cisplatin weekly $\left(30-40 \mathrm{mg} / \mathrm{m}^{2}\right)$ or on weeks 1,4 and $7\left(80-100 \mathrm{mg} / \mathrm{m}^{2}\right)$ of radiotherapy.

\section{Follow-Up and Statistical Analysis}

Follow-up was measured from first day of therapy to last examination or death. Patients were followed by MRI and plasma EBV DNA at least every 3 months during first 2 years, then every 6 months thereafter (or until death). The end points (time to first defining event) were distant metastasis-free survival (DMFS), overall survival (OS), disease-free survival (DFS), locoregional relapse-free survival (LRRFS), and DMFS was set as the primary endpoint.

Propensity scores were computed by logistic regression for each patient using the following covariates: age, gender, concurrent chemotherapy, $\mathrm{T}$-stage, N-stage, overall stage and pre-DNA. Receiver operating characteristic (ROC) curve analysis was used to calculate the cut-off value for pre-treatment EBV DNA. The Chi-square test or Fisher's exact test were used to compare categorical variables and treatment failure patterns between the NCT and non-NCT groups. Life-table estimation was performed using the Kaplan-Meier method and log-rank test. The multivariate Cox proportional hazards model was used to estimate hazard ratios (HRs) and 95\% confidence intervals (CIs); age, gender, smoking, drinking, $\mathrm{T}$ category, $\mathrm{N}$ category, overall stage, concurrent chemotherapy and NCT were included as variables. All tests were two-sided; $P<$ 0.05 was considered significant. Stata Statistical Package 12 (StataCorp LP, College Station, TX, USA) was used for all analyses.

\section{Results}

\section{Cut-off Value of Pre-treatment EBV DNA}

In total, $246 / 1099(22.4 \%)$ patients had undetectable pre-DNA. The median pre-DNA level for the whole cohort was 3400 copies $/ \mathrm{ml}$ (interquartile range, 110-23400). Based on ROC curve analysis, the cut-off value of pre-DNA was 1550 copies/ml for DMFS (area under curve [AUC], 0.655; sensitivity, 0.819; specificity, 0.445). Therefore, patients were classified as high pre-DNA $(\geq 1550$ copies/ml) and low pre-DNA $(<1550$ copies/ml) groups based on this value. Finally, 453 (41.2\%) patients in the low pre-DNA group were further analysed.

Table 1. Baseline characteristics of all 145 pairs of locoregionally advanced NPC patients with pre-DNA $<1550$ copies $/ \mathrm{ml}$.

\begin{tabular}{|c|c|c|c|}
\hline & NCT & Non-NCT & $\mathrm{Pa}$ \\
\hline Characteristics & No. (\%) & No. (\%) & \\
\hline Age (y) & & & 0.607 \\
\hline$<50$ & $100(69.0)$ & 104 (71.7) & \\
\hline$\geq 50$ & $45(31.0)$ & $41(28.3)$ & \\
\hline Gender & & & 0.685 \\
\hline Male & $107(73.8)$ & $110(75.9)$ & \\
\hline Female & $38(26.2)$ & $35(24.1)$ & \\
\hline Smoking & & & 0.905 \\
\hline Yes & 59 (40.7) & $58(40.0)$ & \\
\hline No & $86(59.3)$ & $87(60.0)$ & \\
\hline Drinking & & & 1.000 \\
\hline Yes & $19(13.1)$ & $19(13.1)$ & \\
\hline No & $126(86.9)$ & $126(86.9)$ & \\
\hline Concurrent & & & 0.881 \\
\hline Yes & $118(81.4)$ & 117 (80.7) & \\
\hline No & $27(18.6)$ & $28(19.3)$ & \\
\hline $\mathrm{T}_{\text {category }} \mathrm{b}$ & & & 0.679 \\
\hline $\mathrm{T} 1$ & $8(5.5)$ & $11(7.6)$ & \\
\hline $\mathrm{T} 2$ & $6(4.1)$ & $3(2.0)$ & \\
\hline $\mathrm{T} 3$ & $99(68.3)$ & $98(67.6)$ & \\
\hline $\mathrm{T} 4$ & $32(22.1)$ & $33(22.8)$ & \\
\hline $\mathrm{N}$ category $\mathrm{b}$ & & & 0.563 \\
\hline No & 24 (16.6) & $26(17.9)$ & \\
\hline N1 & $89(61.4)$ & $87(60.0)$ & \\
\hline $\mathrm{N} 2$ & $29(20.0)$ & 25 (17.2) & \\
\hline N3 & $3(2.0)$ & $7(4.9)$ & \\
\hline Overall stage $b$ & & & 0.503 \\
\hline III & 110 (75.9) & 105 (72.4) & \\
\hline IVA-IVB & $35(24.1)$ & 40 (27.6) & \\
\hline
\end{tabular}

\section{Baseline Characteristics and failure patterns}

From the original 453 NPC patients, 145 pairs were selected by PSM (Table 1). No significant difference was found between the NCT and non-NCT groups $(P=0.727)$ with regard to pre-DNA, and other factors were well balanced. The median follow-up duration for the entire cohort was 50.3 months (range, 4.6-68.3 months). Up to the last follow-up, 9 (6.2\%) patients in NCT group and $7(4.8 \%)$ patients in non-NCT group developed local recurrence; 3 (2.1\%) patients in NCT group and $4(2.8 \%)$ patients in non-NCT group experienced regional recurrence; and $4(2.8 \%)$ patients in NCT group and $10(6.9 \%)$ patients in non-NCT group experienced distant metastasis. Moreover, 11 (7.6\%) patients and $12(8.3 \%)$ patients died in NCT and on-NCT group, respectively. 


\section{Prognostic value of NCT}

The 3-year DMFS, OS, DFS and LRRFS rates for the entire cohort were $96.1 \%, 95.1 \%, 89.6 \%$ and $94.7 \%$, respectively. For NCT group vs. non-NCT group, the 3-year DMFS, OS, DFS and LRRFS rates were $98.6 \%$ vs. $93.7 \%(P=0.101$, Figure $1 \mathrm{~A}), 95.8 \%$ vs. $94.4 \%(P=$ 0.881 , Figure $1 \mathrm{~B}), 91.7 \%$ vs. $87.5 \%(P=0.309$, Figure $1 C)$ and $94.4 \%$ vs. $95.0 \%(P=0.667$, Figure $1 D)$, respectively.

Multivariate analysis was performed to adjust for various prognostic factors, and consistent with the results of univariate analysis, it revealed that NCT was not associated with significantly improved DMFS (HR, 0.822; 95\% CI, 0.191-3.532; $P=0.108)$, OS (HR, 0.910; 95\% CI, 0.392-2.114; $P=0.827)$, DFS (HR, 0.657; 95\% CI, 0.343-1.260; $P=0.206$ ) and LRRFS (HR, 0.775; 95\% CI, 0.319-1.882; $P=0.573$; Table 2).

\section{Prognostic value of NCT for patients with stage III and IV}

Patients with stage IV generally exhibited a higher rate of distant metastasis compared with that of patients with stage III NPC. To further explore the prognostic value of NCT in patients with stage III and IV NPC, we conducted a stratified analysis of 110 and 39 PSM-selected pairs of NPC patients with stage III and IV, respectively.

With regard to the patients with stage III NPC, the 3-year DMFS (99.1\% vs. $98.2 \%$, respectively; $P=$
0.343; Figure 2A), OS (98.2\% vs. $97.2 \%$, respectively; $P$ $=0.966$; Figure 2B), DFS $(93.6 \%$ vs. $94.5 \%$, respectively; $P=0.555)$ and LRRFS $(95.4 \%$ vs. $97.2 \%$, respectively; $P=0.850$ ) were comparable between patients receiving NCT and those not receiving NCT. Moreover, for patients with stage IV NPC, NCT also did not significantly improve 3-year DMFS $(91.7 \%$ vs. $81.3 \%$, respectively; $P=0.357$; Figure $2 \mathrm{C})$, OS $(84.6 \%$ vs. $87.1 \%$, respectively; $P=0.822$; Figure $2 \mathrm{D})$, DFS (76.9\% vs. $71.7 \%$, respectively; $P=0.930)$ and LRRFS $(89.0 \%$ vs. $92.0 \%$, respectively; $P=0.969)$.

Table 2. Multivariate analysis of prognostic factors for all 145 pairs of locoregionally advanced NPC patients with pre-DNA < 1550 copies $/ \mathrm{ml}$.

\begin{tabular}{llll}
\hline Endpoints & Variable & HR $(95 \% \mathrm{CI})$ & $\mathrm{Pa}$ \\
\hline DMFS & NCT & $0.822(0.191-3.532)$ & 0.108 \\
& Overall stage & $8.849(2.409-32.502)$ & 0.001 \\
OS & NCT & $0.910(0.392-2.114)$ & 0.827 \\
& Overall stage & $5.366(2.266-12.707)$ & $<0.001$ \\
& Age & $2.807(1.226-6.428)$ & 0.015 \\
DFS & NCT & $0.657(0.343-1.260)$ & 0.206 \\
& Overall stage & $3.125(1.652-5.914)$ & $<0.001$ \\
& Age & $2.189(1.156-4.145)$ & 0.016 \\
LRRFS & NCT & $0.775(0.319-1.882)$ & 0.573 \\
\hline
\end{tabular}

Abbreviations: NPC $=$ nasopharyngeal carcinoma; pre-DNA $=$ pre-treatment Epstein-Barr virus DNA; $\mathrm{HR}$ = hazard ratio; $\mathrm{CI}=$ confidence interval; DMFS distant metastasis-free survival; OS = overall survival; DFS = disease-free survival; LRRFS = locoregional relapse-free survival; NCT = neoadjuvant chemotherapy. a: Multivariate $P$-values were calculated using an adjusted Cox proportional-hazards model with backward elimination and the following parameters: age ( $\geq 50 \mathrm{y}$ vs. $<50 \mathrm{y}$ ), gender (male or female), smoking (yes or no), drinking (yes or no), T category (T1-2 or T3-4), N category (N0-1 or N2-3), concurrent chemotherapy (yes or no) and NCT (yes or No).
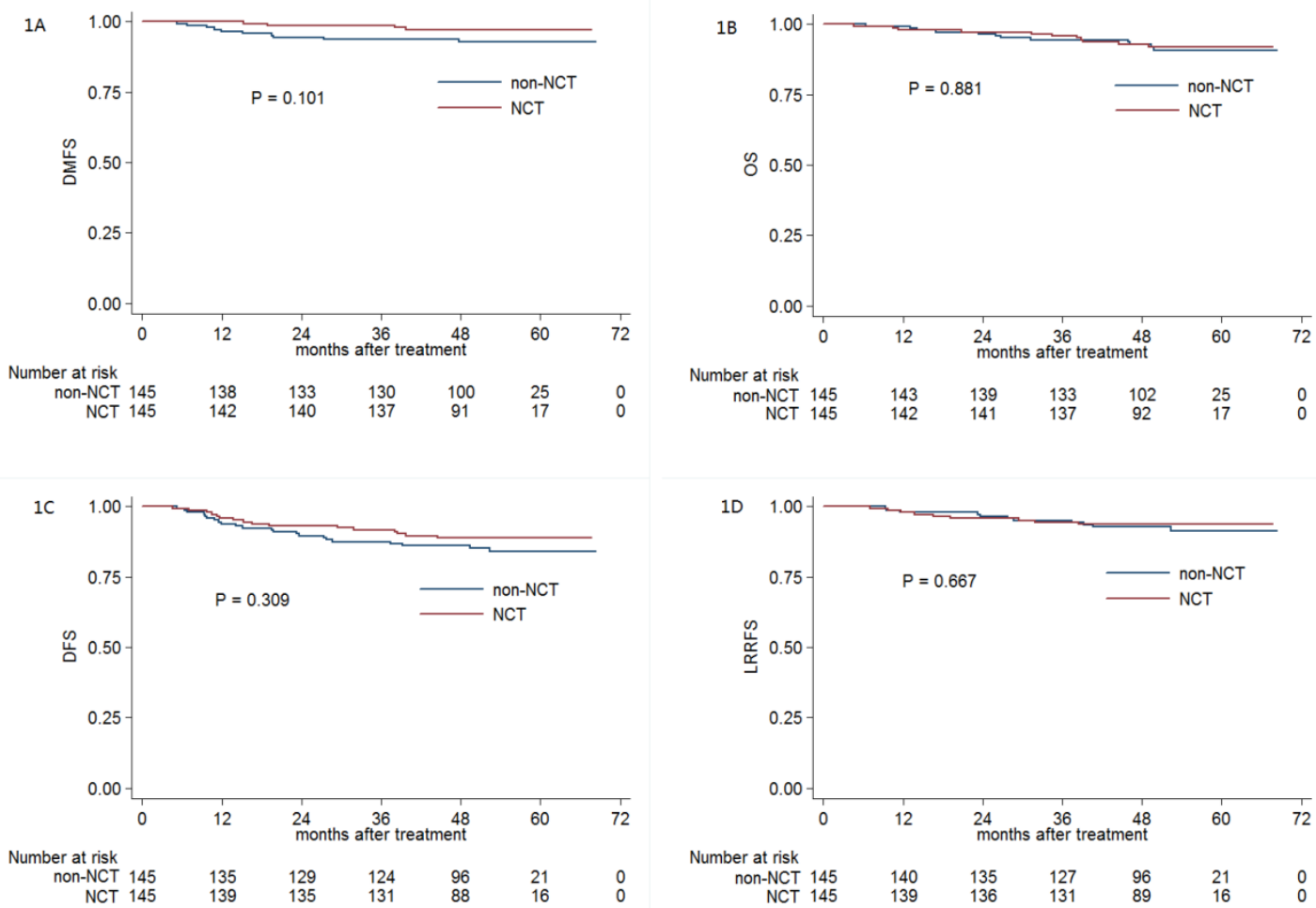

Figure 1. Kaplan-Meier DMFS (A), OS (B), DFS (C) and LRRFS (D) curves for NCT and non-NCT patients with pre-DNA < 1550 copies/ml. Abbreviations: DMFS = distant metastasis-free survival; OS = overall survival; DFS = disease-free survival; LRRFS = locoregional relapse-free survival; NCT = neoadjuvant chemotherapy; pre-DNA = pre-treatment Epstein-Barr virus DNA. 

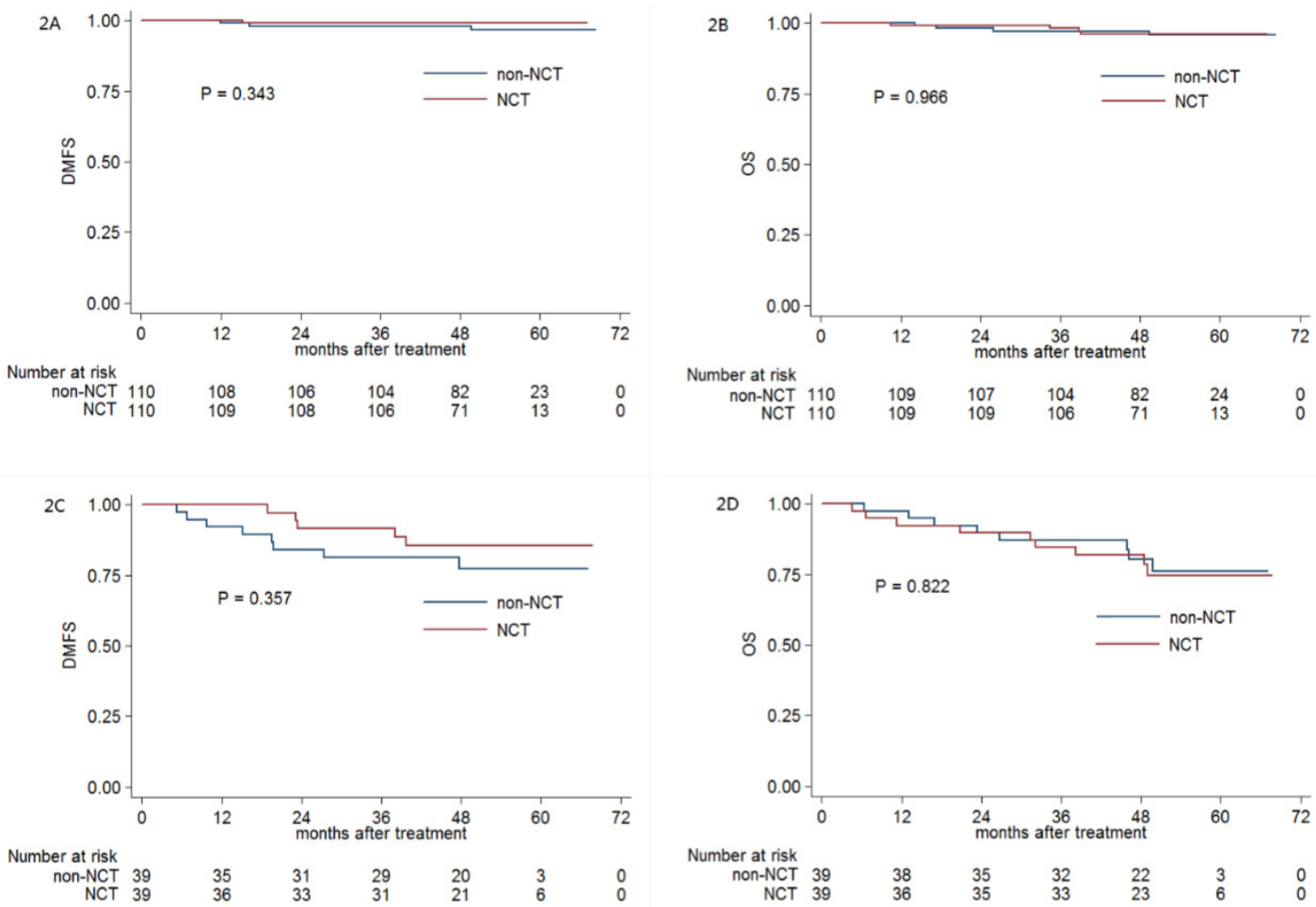

Figure 2. Kaplan-Meier DMFS (A) and OS (B), DMFS (C) and OS (D) curves for NCT and non-NCT patients stratified as stage III and IV, respectively. Abbreviations: DMFS = distant metastasis-free survival; OS = overall survival; DFS = disease-free survival; LRRFS = locoregional relapse-free survival; NCT = neoadjuvant chemotherapy.

\section{Prognostic value of NCT for patients with N0-1 and N2-3 category}

Obviously, advanced $\mathrm{N}$ stage was associated with significant higher distant metastasis rate compared with early $\mathrm{N}$ category. Therefore, we conducted this subgroup analysis to further investigate the efficacy of NCT in NPC patients with early (N0-1) and advanced (N2-3) category; 111 and 32 pairs of NPC patients were respectively selected by PSM.

In early $\mathrm{N}$ category group, the 3-year DMFS (98.2\% vs. $93.6 \%$, respectively; $P=0.232$; Figure $3 \mathrm{~A}$ ), OS $(95.5 \%$ vs. $95.5 \%$, respectively; $P=0.649$; Figure 3B), DFS (91.0\% vs. $88.2 \%$, respectively; $P=0.689)$ and LRRFS ( $92.7 \%$ vs. $94.5 \%$, respectively; $P=0.80)$ were comparable between patients receiving or not receiving NCT. In advanced $\mathrm{N}$ category group, no significant difference of 3-year DMFS (100\% vs. $93.5 \%$, respectively; $P=0.157$; Figure $3 C)$, OS (93.8\% vs. $90.6 \%$, respectively; $P=0.984$; Figure 3D), DFS $(90.6 \%$ vs. $90.6 \%$, respectively; $P=0.994)$ and LRRFS $(96.7 \%$ vs. $100 \%$, respectively; $P=0.326$ ) was found between patients receiving or not receiving NCT.

\section{Discussion}

To the best of our knowledge, this is the first study to investigate the clinical efficacy of NCT in locoregionally advanced NPC with low pre-treatment
EBV DNA in the era of IMRT. By using PSM and multivariate analysis, this study provided the fairest comparisons of matched patients and revealed NCT was not associated with significantly improved prognosis. Moreover, further stratified analysis based on overall stage and $\mathrm{N}$ category also failed to show the efficacy of NCT in reduce distant metastasis.

In the era of IMRT, distant metastasis has been the main treatment failure pattern [6]. Plenty of previous studies have tried to figure out the prognostic value of NCT in locoregionally advanced NPC [7-12]; however, these results were controversial. Except for the factor that different chemotherapy regimens were used, the negative results may be attributed to the reason that many patients recruited in these trials had low risk of distant metastasis and did not really benefit from NCT. A positive effect on progression-free survival (PFS) was reported in stage IV NPC with N2-3 category in comparing NCT plus RT with RT alone [10], which indicated that only highly selected patients would benefit from NCT. In the study by $\mathrm{Du}$ et al. [20], advanced $\mathrm{N}$ category (N2-3), pre-DNA (> 4000 copies/ml), serum albumin $(\leq 46 \mathrm{~g} / \mathrm{L})$ and platelet count $(>300 \mathrm{k} / \mathrm{cc})$ were used to define high-risk patients who may benefit from NCT. Therefore, NCT should be effective in high-risk locoregionally advanced NPC. 

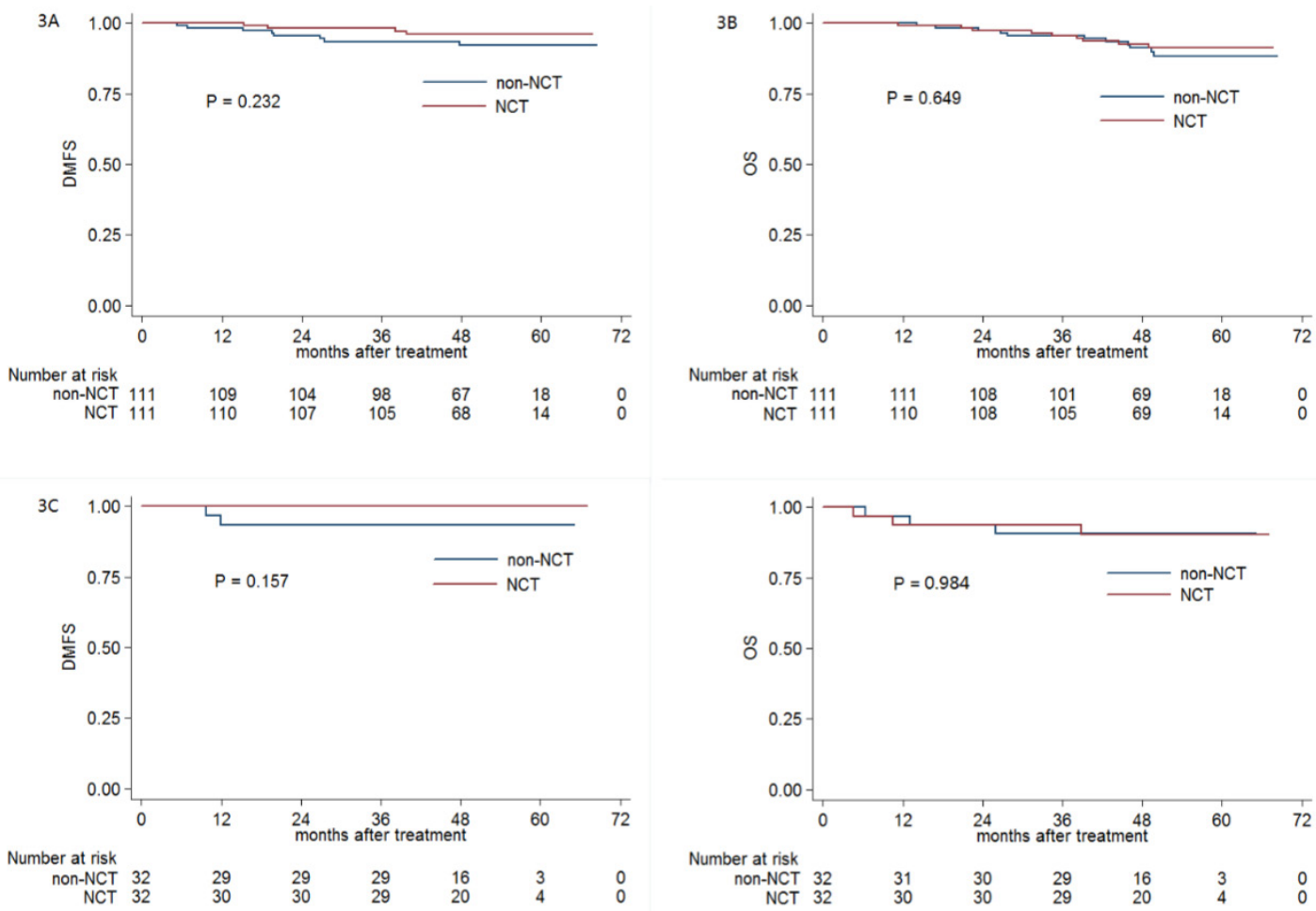

Figure 3. Kaplan-Meier DMFS (A) and OS (B), DMFS (C) and OS (D) curves for NCT and non-NCT patients stratified as N0-1 and N2-3 category, respectively. Abbreviations: DMFS = distant metastasis-free survival; OS = overall survival; DFS = disease-free survival; LRRFS = locoregional relapse-free survival; NCT = neoadjuvant chemotherapy.

High pre-DNA could accurately predict distant metastasis [18, 24]. All the patients recruited for this study had a pre-DNA < 1550 copies/ml which was defined as low-risk distant metastasis, and the outcomes of this current study suggested NCT prior to RT could not improve DMFS in low-risk locoregionally advanced NPC. In order to select the optimal patients who could really benefit from NCT, stratified analysis based on overall stage and $\mathrm{N}$ category was performed. However, the results of subgroup analysis still demonstrated no significant survival benefit with the additional using of NCT. These negative results should be attributed to two possible reasons. Firstly, NCT did not have the potential value of reducing distant metastasis. Secondly, the relatively small sample in each subgroup resulted in low test power and the prognostic difference between the two groups could not be detected.

In this study, we used only pre-DNA as the biomarker to define locoregionally advanced NPC with low-risk distant metastasis because plasma EBV DNA has been widely reported and proven to be a reliable factor in predicting prognosis [14-18]. Although many other prognostic factors as primary tumor volume [25] and pre-treatment serum lactate dehydrogenase (LDH) $[26,27]$ have been reported to correlate with distant metastasis; these studies were retrospective and no prospective clinical trials were conducted to confirm the results. Therefore, we did not take these factors into consideration. Moreover, we only analyzed the prognostic value of NCT in patients with low-pre-treatment EBV DNA because the study by $\mathrm{Du}$ et al. had proven that NCT was effective in patients with high pre-treatment EBV DNA. The results of this study could somehow complement the results in abovementioned studies $[10,20]$.

In conclusion, our outcomes suggested that NCT was not feasible and effective in locoregionally advanced NPC with low pre-DNA in the era of IMRT. Therefore, at clinical practice, NCT should not be delivered for highly selected low-risk patients with locoregionally advanced NPC. One main limitation of this study is that our study was a retrospective analysis of patients who were treated at a single center. In addition, the sample was relatively small and it may affect the results of stratified analysis. Therefore, further studies consisted of large cohorts are warranted to confirm these results. Moreover, pre-DNA should be considered in selecting eligible patients when conducting prospective clinical trial.

\section{Conclusion}

The prognostic value of NCT was limited, and NCT should not be used in clinical practice for 
locoregionally advanced NPC patients with low pre-treatment EBV DNA in the era of IMRT. Further prospective studies are warranted to confirm these results.

\section{Abbreviations}

NPC: nasopharyngeal carcinoma; RT: radiotherapy; CCRT: concurrent chemoradiotherapy; NCCN: national comprehensive cancer network; IMRT: intensity-modulated radiotherapy; NCT: neoadjuvant chemotherapy; EBV: Epstein-Barr virus; Pre-DNA: pre-treatment Epstein-Barr virus DNA; PSM: propensity score matching; WHO: World Health Organization; MRI: magnetic resonance imaging; PET: positron emission tomography; IgA: immunoglobulin A; VCA: viral caspid antigen; EA: early antigen; UICC/AJCC: International Union against Cancer/American Joint Committee on Cancer; PTV: planning target volume; GTVnx: gross tumour volume; GTVnd: gross tumour volume of the involved lymph nodes; CTV1: high-risk clinical target volume; CTV2: low-risk clinical target volume; ACT: adjuvant chemotherapy; DMFS: distant metastasis-free survival; OS: overall survival; DFS: disease-free survival; LRRFS: locoregional relapse-free survival; ROC: receiver operating characteristic; HRs: hazard ratios; Cis: confidence intervals; AUC: area under curve; PFS: progression-free survival; $\mathrm{LDH}$ : lactate dehydrogenase.

\section{Acknowledgments}

This work was supported by grants from the Health \& Medical Collaborative Innovation Project of Guangzhou City, China (No. 201400000001), the Sun Yat-sen University Clinical Research 5010 Program (No. 2012011), the Science and Technology Project of Guangzhou City, China (No. 14570006), and the Planned Science and Technology Project of Guangdong Province, China (No. 2013B020400004).

\section{Competing Interests} interests.

The authors declare no competing financial

\section{References}

1. Jemal A, Bray F, Center MM, et al. Global cancer statistics. CA Cancer J Clin. 2011; 61: 69-90.

2. Al-Sarraf M, LeBlanc M, Giri PG, et al. Chemoradiotherapy versus radiotherapy in patients with advanced nasopharyngeal cancer: phase III randomized Intergroup study 0099. J Clin Oncol. 1998; 16: 1310-7.

3. Lee AW, Lau WH, Tung SY, et al. Preliminary results of a randomized study on therapeutic gain by concurrent chemotherapy for regionally-advanced nasopharyngeal carcinoma: NPC-9901 Trial by the Hong Kong Nasopharyngeal Cancer Study Group. J Clin Oncol. 2005; 23: 6966-75.

4. Lin JC, Jan JS, Hsu CY, et al. Phase III study of concurrent chemoradiotherapy versus radiotherapy alone for advanced nasopharyngeal carcinoma: positive effect on overall and progression-free survival. J Clin Oncol. 2003; 21: 631-7.

5. Wee J, Tan EH, Tai BC, et al. Randomized trial of radiotherapy versus concurrent chemoradiotherapy followed by adjuvant chemotherapy in patients with American Joint Committee on Cancer/International Union against cancer stage III and IV nasopharyngeal cancer of the endemic variety. J Clin Oncol. 2005; 23: 6730-8.

6. Sun $\mathrm{X}, \mathrm{Su} \mathrm{S}, \mathrm{Chen} \mathrm{C}$, et al. Long-term outcomes of intensity-modulated radiotherapy for 868 patients with nasopharyngeal carcinoma: an analysis of survival and treatment toxicities. Radiother Oncol. 2014; 110: 398-403.

7. Chua DT, Sham JS, Choy D, et al. Preliminary report of the Asian-Oceanian Clinical Oncology Association randomized trial comparing cisplatin and epirubicin followed by radiotherapy versus radiotherapy alone in the treatment of patients with locoregionally advanced nasopharyngeal carcinoma. Asian-Oceanian Clinical Oncology Association Nasopharynx Cancer Study Group. Cancer. 1998; 83: 2270-83.

8. Hong RL, Ting LL, Ko JY, et al. Induction chemotherapy with mitomycin, epirubicin, cisplatin, fluorouracil, and leucovorin followed by radiotherapy in the treatment of locoregionally advanced nasopharyngeal carcinoma. J Clin Oncol. 2001; 19: 4305-13.

9. Hui EP, Ma BB, Leung SF, et al. Randomized phase II trial of concurrent cisplatin-radiotherapy with or without neoadjuvant docetaxel and cisplatin in advanced nasopharyngeal carcinoma. J Clin Oncol. 2009; 27: 242-9.

10. International Nasopharynx Cancer Study G, Trial VI. Preliminary results of a randomized trial comparing neoadjuvant chemotherapy (cisplatin, epirubicin, bleomycin) plus radiotherapy vs. radiotherapy alone in stage $\operatorname{IV}(>$ or $=\mathrm{N} 2$, M0) undifferentiated nasopharyngeal carcinoma: a positive effect on progression-free survival. Int J Radiat Oncol Biol Phys. 1996; 35: 463-9.

11. Ma J, Mai $\mathrm{HQ}$, Hong $\mathrm{MH}$, et al. Results of a prospective randomized trial comparing neoadjuvant chemotherapy plus radiotherapy with radiotherapy alone in patients with locoregionally advanced nasopharyngeal carcinoma. J Clin Oncol. 2001; 19: 1350-7.

12. Tan T, Lim WT, Fong KW, et al. Concurrent chemo-radiation with or without induction gemcitabine, Carboplatin, and Paclitaxel: a randomized, phase 2/3 trial in locally advanced nasopharyngeal carcinoma. Int J Radiat Oncol Biol Phys. 2015; 91: 952-60.

13. OuYang PY, Xie C, Mao YP, et al. Significant efficacies of neoadjuvant and adjuvant chemotherapy for nasopharyngeal carcinoma by meta-analysis of published literature-based randomized, controlled trials. Ann Oncol. 2013; 24: 2136-46.

14. An X, Wang FH, Ding PR, et al. Plasma Epstein-Barr virus DNA level strongly predicts survival in metastatic/recurrent nasopharyngeal carcinoma treated with palliative chemotherapy. Cancer. 2011; 117: 3750-7.

15. Lin JC, Wang WY, Chen KY, et al. Quantification of plasma Epstein-Barr virus DNA in patients with advanced nasopharyngeal carcinoma. N Engl J Med. 2004; 350: 2461-70.

16. Lin JC, Wang WY, Liang WM, et al. Long-term prognostic effects of plasma epstein-barr virus DNA by minor groove binder-probe real-time quantitative PCR on nasopharyngeal carcinoma patients receiving concurrent chemoradiotherapy. Int J Radiat Oncol Biol Phys. 2007; 68: 1342-8.

17. Lo YM, Chan LY, Chan AT, et al. Quantitative and temporal correlation between circulating cell-free Epstein-Barr virus DNA and tumor recurrence in nasopharyngeal carcinoma. Cancer Res. 1999; 59: 5452-5.

18. Peng H, Guo R, Chen L, et al. Prognostic Impact of Plasma Epstein-Barr Virus DNA in Patients with Nasopharyngeal Carcinoma Treated using Intensity-Modulated Radiation Therapy. Sci Rep. 2016; 6: 22000.

19. Peng $\mathrm{H}$, Chen L, Zhang $\mathrm{Y}$, et al. Survival analysis of patients with advanced-stage nasopharyngeal carcinoma according to the Epstein-Barr virus status. Oncotarget 2016; [Epub ahead of print].

20. Du XJ, Tang LL, Chen L, et al. Neoadjuvant chemotherapy in locally advanced nasopharyngeal carcinoma: Defining high-risk patients who may benefit before concurrent chemotherapy combined with intensity-modulated radiotherapy. Sci Rep. 2015; 5: 16664 .

21. Austin PC. The relative ability of different propensity score methods to balance measured covariates between treated and untreated subjects in observational studies. Med Decis Making. 2009; 29: 661-77.

22. Edge SB, Compton CC. The American Joint Committee on Cancer: the 7th edition of the AJCC cancer staging manual and the future of TNM. Ann Surg Oncol. 2010; 17: 1471-4.

23. Shao JY, Zhang Y, Li YH, et al. Comparison of Epstein-Barr virus DNA level in plasma, peripheral blood cell and tumor tissue in nasopharyngeal carcinoma. Anticancer Res. 2004; 24: 4059-66.

24. Tang LQ, Chen QY, Fan W, et al. Prospective study of tailoring whole-body dual-modality [18F]fluorodeoxyglucose positron emission tomography/computed tomography with plasma Epstein-Barr virus DNA for detecting distant metastasis in endemic nasopharyngeal carcinoma at initial staging. J Clin Oncol. 2013; 31: 2861-9.

25. Guo R, Sun $Y, Y u$ XL, et al. Is primary tumor volume still a prognostic factor in intensity modulated radiation therapy for nasopharyngeal carcinoma? Radiother Oncol. 2012; 104: 294-9.

26. Wan $\mathrm{XB}, \mathrm{Wei} \mathrm{L}, \mathrm{Li} \mathrm{H}$, et al. High pretreatment serum lactate dehydrogenase level correlates with disease relapse and predicts an inferior outcome in locally advanced nasopharyngeal carcinoma. Eur J Cancer. 2013; 49: 2356-64.

27. Zhou GQ, Ren XY, Mao YP, et al. Prognostic implications of dynamic serum lactate dehydrogenase assessments in nasopharyngeal carcinoma patients treated with intensity-modulated radiotherapy. Sci Rep. 2016; 6: 22326. 